\title{
Oculomaxillofacial dysostosis
}

INSERM

\section{Source}

INSERM. (1999). Orphanet: an online rare disease and orphan drug data base.

Oculomaxillofacial dysostosis. ORPHA:1794

Oculomaxillofacial dysostosis is a rare, genetic bone developmental disorder characterized by short stature, orbital region and ocular abnormalities (e.g. asymmetric orbits, anophthalmia, down-slanted and S-shaped palpebral fissures, sparse eyebrows/eyelashes, abnormal eyelids, ectropion, symblepharon, corneal leukoma), abnormal nose (e.g. broad and abnormally modeled nasal root, bridge and tip, lateral deviation), malar hypoplasia, cleft lip/palate, and oblique facial clefts. Intellectual disability, microcephaly, micrognathia and limb anomalies (e.g. hemimelia, abnormal scapular girdle, brachydactyly, syndactyly, broad halluces) have also been reported. 